\title{
Ratio of the vitreous vascular endothelial growth factor and pigment epithelial-derived factor in Eales disease
}

\author{
Narayanasamy Angayarkanni • Radhakrishnan Selvi • \\ Rishi Pukhraj • Jyotirmoy Biswas • Shah J. Bhavesh • \\ Joyce Tombran-Tink
}

Received: 13 November 2008 / Accepted: 16 February 2009 / Published online: 6 March 2009

(C) The Author(s) 2009. This article is published with open access at Springerlink.com

\begin{abstract}
Eales disease (ED) is an idiopathic inflammatory venous occlusion of the peripheral retina. As neovascularization is prominent in $\mathrm{ED}$, this study attempts to look at the ratio of VEGF, the angiogenic factor, and PEDF, an anti-angiogenic factor in the vitreous of ED patients in comparison with the macular hole $(\mathrm{MH})$ and Proliferative Diabetic Retinopathy (PDR). Vitreous levels of VEGF and PEDF were determined in the undiluted vitreous specimen obtained from $26 \mathrm{ED}$ cases, 17 PDR, and seven patients with $\mathrm{MH}$. The vitreous levels of VEGF and PEDF were estimated by ELISA. The immunohistochemistry (IHC) for VEGF and PEDF were done in the epiretinal membrane of ED and PDR case. The VEGF/PEDF ratio was found to be significantly increased in $\operatorname{ED}(p=0.014)$ and $\operatorname{PDR}(p=$ $0.000)$ compared to $\mathrm{MH}$. However the ratio was 3.5-fold higher in PDR than ED $(p=0.009)$. The IHC data on the ERM specimen from ED showed the presence of VEGF
\end{abstract}

N. Angayarkanni $(\bowtie) \cdot R$. Selvi

Biochemistry Research Department, Sankara Nethralaya,

Vision Research Foundation,

18, College Road,

Chennai 600 006, India

e-mail: drak@snmail.org

R. Pukhraj · S. J. Bhavesh

Sri Bhagvan Mahavir Vitreo-Retinal Services,

Medical Research Foundation,

Kolkata, India

J. Biswas

Ocular pathology, Medical Research Foundation,

Kolkata, India

J. Tombran-Tink

Department of Ophthalmology and Visual science,

Yale University,

New Haven, CT, USA and PEDF similar to PDR. The high angiogenic potential seen as the ratio of VEGF/PEDF correlates with the peak clinical onset of the disease in the age group 21-30 years and the diseases usually self-resolves above the age of 40 , which is reflected by the low ratio of VEGF/PEDF. The study shows that the VEGF/PEDF ratio is significantly increased in ED though the angiogenic potential is higher in PDR than in ED. Clinically Eales Disease is known as a self-limiting disease, while PDR is a progressive disease.

Keywords VEGF. PEDF. Ratio - Eales disease ·

Proliferative diabetic retinopathy

\section{Introduction}

Eales disease (ED) is an idiopathic inflammatory venous occlusion that primarily affects the peripheral retina of adults. Young adult males have been reported to have an increased prevalence, with the peak age of onset as 2035 years and a reported range of 13-63 years [1, 2]. However, a study of 55 patients by Gieser et al. showed that men and women were affected equally [3].

ED has been reported from the UK, USA, and Canada in the later half of nineteenth and early twentieth centuries. No racial predilection is known in ED. However, the disease is more prevalent in India and portions of the Middle East. The reported incidence in India is one in 200 to 250 ophthalmic patients [1]. The number of new cases per year in one of the referral hospital in India where this study was conducted (Sankara Nethralaya, Chennai, India) for the last 10 years shows an average of 310 fresh patients per year. Most patients present with symptoms of floaters, specks, cobwebs, blurring, or decreased vision associated with vitreous 
hemorrhage. Often, patients complain of uniocular symptoms, but ophthalmic examination reveals early changes of ED in the other eye. Bilateral involvement is evident in $80-90 \%$ of patients. The major manifestations of ED are inflammation, neovascularization, vitreous hemorrhage, and retinal detachment. Bilateral involvement is evident in $80-90 \%$ of the patients. There is no known mortality associated with ED. Stages of ED broadly include stage of retinal phlebitis, stage of peripheral nonperfusion, and stage of retinal neovascularization [4]. A new classification system has been proposed for ED as Stage 1: periphlebitis of small (1a) and large (1b) caliber vessels with superficial retinal hemorrhages; Stage 2a: capillary nonperfusion, Stage 2b: neovascularization elsewhere/of the disc; Stage 3a: fibrovascular proliferation, Stage 3b: vitreous hemorrhage; Stage 4a: traction/combined rhegmatogenous retinal detachment, and Stage $4 \mathrm{~b}$ : rubeosis iridis, neovascular glaucoma, complicated cataract, and optic atrophy [5].

The etiopathogenesis of this disease remains obscure since its description. Association of ED with several systemic diseases, mainly tuberculosis, has been reported. However, in large series, many such associations have not been proven. Several reports have shown association of a variety of neurological and hematological disorders with ED. Immune-mediated mechanism has been proposed by many authors. However, so far, a precise immunological mechanism has not been identified [4].

The histopathological hallmark in ED is the adhesion of leukocytes to the endothelium and the infiltration of these cells into the retinal parenchyma. Phagocytegenerated free radicals have been implicated in mediating tissue damage associated with various inflammatory vasculopathies. Our earlier studies show elevated levels of reactive oxygen species and reactive nitrogen species products correlating with diminished antioxidant status in patients with ED, and based on these studies, increased oxidative stress has been strongly implicated in the etiopathogenesis of ED [2, 6-8].

Intraocular neovascularization occurs in numerous ischemic retinal disorders, including diabetic retinopathy, ischemic retinal-vein occlusion, and retinopathy of prematurity. This

Table 1 Clinical details of the Eales disease cases

\begin{tabular}{|c|c|c|c|c|c|c|c|}
\hline S. No. & Age/Sex & $\mathrm{OD} / \mathrm{OS}$ & Classification & Quadrangles & Steroid & Laser & SystemicIllness \\
\hline 1 & $26 / \mathrm{M}$ & OD & $3 b$ & 4 & No & Not done & Nil \\
\hline 2 & $30 / \mathrm{M}$ & OS & $3 b$ & 4 & No & Sectoral PHC & Nil \\
\hline 3 & $19 / \mathrm{M}$ & OD & $3 b$ & 4 & Yes & Sectoral PHC & Nil \\
\hline 4 & $35 / \mathrm{M}$ & OD & $3 b$ & 4 & No & Sectoral PHC & Nil \\
\hline 5 & $25 / \mathrm{M}$ & OD & $3 \mathrm{a}$ & 4 & No & PRP & Nil \\
\hline 6 & 39/M & OS & $3 b$ & 4 & No & Sectoral PHC & Nil \\
\hline 7 & $33 / \mathrm{M}$ & OD & $3 b$ & 4 & No & Not done & Nil \\
\hline 8 & $29 / \mathrm{M}$ & OS & $3 b$ & 4 & No & Not done & Nil \\
\hline 9 & $36 / \mathrm{M}$ & OS & $4 a$ & 4 & No & Not done & HT \\
\hline 10 & $30 / \mathrm{M}$ & OD & $3 b$ & 4 & No & PRP & Nil \\
\hline 11 & $33 / \mathrm{M}$ & OD & $4 a$ & 4 & Yes & PRP & Nil \\
\hline 12 & $14 / \mathrm{M}$ & OD & $3 b$ & 4 & Yes & PRP & Nil \\
\hline 13 & $51 / \mathrm{F}$ & OD & $3 b$ & 4 & No & PRP & Nil \\
\hline 14 & $36 / \mathrm{M}$ & OS & $3 b$ & 4 & No & PRP & Nil \\
\hline 15 & $24 / \mathrm{M}$ & OS & $3 b$ & 4 & No & PRP & Nil \\
\hline 16 & $28 / \mathrm{M}$ & OD & $4 a$ & 4 & No & Not done & Nil \\
\hline 17 & $45 / \mathrm{M}$ & OS & $3 b$ & 4 & No & Not done & Nil \\
\hline 18 & $35 / \mathrm{M}$ & OD & $3 b$ & 4 & No & Not done & Nil \\
\hline 19 & $34 / \mathrm{M}$ & OS & $3 b$ & 4 & Yes & Not done & HT \\
\hline 20 & $43 / \mathrm{M}$ & OS & $4 a$ & 4 & No & Not done & Nil \\
\hline 21 & $33 / \mathrm{M}$ & OS & $3 b$ & 4 & No & PRP & Nil \\
\hline 22 & $38 / \mathrm{M}$ & OS & $4 a$ & 4 & No & Not done & Nil \\
\hline 23 & $23 / \mathrm{M}$ & OS & $3 b$ & 4 & No & PRP & Nil \\
\hline 24 & $33 / \mathrm{M}$ & OS & $4 a$ & 4 & No & PRP & Nil \\
\hline 25 & $36 / \mathrm{M}$ & OS & $3 b$ & 4 & No & Not done & Nil \\
\hline 26 & $36 / \mathrm{M}$ & OS & $3 b$ & 4 & No & Not done & Nil \\
\hline
\end{tabular}


Table 2 Clinical details of the proliferative diabetic retinopathy cases

\begin{tabular}{llllllr}
\hline S. No. & Age/sex & OD/OS & Type of DM & Laser & Systemic complications & Diabetes duration \\
\hline 1 & $52 / \mathrm{M}$ & OS & 1 & PRP & HT, CRF & 19 \\
2 & $60 / \mathrm{M}$ & OD & 2 & Not done & Nil & 15 \\
3 & $58 / \mathrm{M}$ & OS & 2 & Not done & Nil & 11 \\
4 & $50 / \mathrm{M}$ & OS & 2 & PRP & CRF & 6 \\
5 & $50 / \mathrm{F}$ & OS & 2 & PRP & HT, CRF & 5 \\
6 & $59 / \mathrm{M}$ & OS & 2 & PRP & HT & 24 \\
7 & $42 / \mathrm{F}$ & OS & 1 & PRP & HT, CRF & 15 \\
8 & $61 / \mathrm{M}$ & OS & 2 & PRP & HT & 15 \\
9 & $43 / \mathrm{F}$ & OD & 1 & PRP & HT, CRF, dyslipidemia & 3 \\
10 & $38 / \mathrm{M}$ & OD & 2 & Not done & Nil & 10 \\
11 & $57 / \mathrm{F}$ & OS & 2 & Not done & Dyslipidemia & 7 \\
12 & $52 / \mathrm{M}$ & OS & 2 & Not done & Nil & 8 \\
13 & $67 / \mathrm{F}$ & OD & 2 & Not done & HT & 6 \\
14 & $42 / \mathrm{M}$ & OD & 1 & Not done & Nil & 10 \\
15 & $65 / \mathrm{M}$ & OS & 2 & PRP & HT & 7 \\
16 & $61 / \mathrm{M}$ & OS & 2 & PRP & HT & 16 \\
17 & $55 / \mathrm{F}$ & OS & 2 & Not done & HT & \\
\hline
\end{tabular}

proliferation often results in vitreous hemorrhage, retinal detachment, and endothelial-cell-specific angiogenic and vasopermeable factor, namely, VEGF, an ischemia-induced ocular angiogenic factor that has an important role in mediating the neovascular response of diabetic retinopathy and other ischemic retinal disorders [9]. Pigment epithelium-derived growth factor (PEDF) is a glycoprotein and a potent inhibitor of ischemia induced neovascularization. Decrease in PEDF is reported in the vitreous of the proliferative diabetic retinopathy patients $[10,11]$.

As neovascularization is prominent in ED this study attempts to look at these two predominant factors one acting as pro- and the other as anti-angiogenic, by estimating the VEGF and PEDF levels, respectively, in the vitreous specimen of ED patients and compare it with that of the PDR which also involves neovascularization and with $\mathrm{MH}$ which does not involve the same. PDR is characterized by presence of newly formed blood vessels arising from the optic disc (NVD) or elsewhere on the surface of retina (NVE). These new vessels may be flat along the surface or elevated into the vitreous cavity and may be with or without a component of glial cell proliferation, fibrovascular proliferation (FVP). As the fibrous tissue contracts, the eyes develop recurrent preretinal and vitreous hemorrhages, tractional retinal detachment, and is occasionally combined with rhegmatogenous retinal detachment. A macular hole is a fullthickness defect of retinal tissue involving the anatomic fovea, thereby affecting central visual acuity [12]. The effect of clinical intervention in terms of the steroid and laser therapy given on the levels of these factors has also been looked into.

\section{Materials and methods}

The study was approved by the Institutional Ethics committee. Undiluted vitreous samples $(0.3-0.5 \mathrm{ml})$ were harvested from the midvitreous at the start of vitrectomy. Vitreous fluid samples were obtained from 26 ED patients (25 M, 1 F, $33 \pm 8$ years), 17 PDR (11 M, 6 F, $54 \pm 9$ years), and seven patients with macular hole ( $3 \mathrm{M}, 4 \mathrm{~F} ; 60 \pm 10$ years). Samples from eyes obtained during repeat vitrectomy were excluded. All the patients were proved clinically by ophthalmologists based on fundus examination by indirect ophthalmoscopy. As far as the diagnosis of ED is concerned, initially, the patient presents with retinal perivasculitis predominantly affecting the peripheral retina (inflammatory stage), then sclerosis of retinal veins indicating retinal ischemia (ischemic stage), and finally retinal or optic disk neovascularization, recurrent vitreous hemorrhage with or without retinal detachment (proliferative stage).

All patients with idiopathic, full-thickness retinal defect of more than $400 \mu \mathrm{m}$ and with posterior vitreous detachment

Table 3 Clinical details of macular hole cases

\begin{tabular}{lllll}
\hline S. No. & Age/sex & OD/OS & Laser & Systemic complications \\
\hline 1 & $68 / \mathrm{M}$ & OD & Not done & IHD \\
2 & $41 / \mathrm{M}$ & OD & Not done & DM \\
3 & $62 / \mathrm{F}$ & OS & Not done & HT \\
4 & $58 / \mathrm{F}$ & OD & Not done & DM \\
5 & $69 / \mathrm{M}$ & OD & Not done & DM \\
6 & $52 / \mathrm{F}$ & OD & Not done & Nil \\
7 & $62 / \mathrm{F}$ & OD & Not done & HT \\
\hline
\end{tabular}


Table 4 Vitreous levels of VEGF in MH, ED, and PDR

VEGF is expressed as mean $\pm \mathrm{SD}$. $P$ values are expressed based on the comparison of the individual disease with macular hole

\begin{tabular}{llll}
\hline Particulars & Macular hole & Eales disease & PDR \\
\hline No. of specimen & 7 & 26 & 17 \\
Mean age (year) & $59 \pm 10(\mathrm{M}-3, \mathrm{~F}-4)$ & $32 \pm 8(\mathrm{M}-25, \mathrm{~F}-1)$ & $54 \pm 8(\mathrm{M}-11, \mathrm{~F}-6)$ \\
Mean VEGF (pg/ml) & $14.9 \pm 9.25$ & $850 \pm 1,832 p=0.000$ & $1,001 \pm 1,165 p=0.001$ \\
Median & 13.18 & 218 & 327 \\
\hline
\end{tabular}

(Stage 4 Idiopathic macular hole; FTMH) were included in the study for the vitreous specimen as the disease control. Careful slit lamp biomicroscopy is usually sufficient to establish the diagnosis in the majority of cases. The WatzkeAllen test is also extremely useful in differentiating FTMHs from other lesions. Optical coherence tomography is used to allow detailed cross-sectional examination of macular holes and may be effective in distinguishing them from other lesions where doubt exists [13].

All cases of types 1 or 2 diabetes mellitus with ocular signs namely, NVE, NVD, FVP, vitreous hemorrhage, tractional retinal detachment, or combined retinal detachment were included in the study for the vitreous specimen from the PDR cases.

Systemic steroid is given to the ED cases in the form of prednisolone, $1 \mathrm{mg} / \mathrm{kg}$ bodyweight given orally and is gradually tapered by $10 \mathrm{mg} /$ week. Tables 1,2 , and 3 show the clinical details of the patients in the study.

Sample collection Vitreous specimens were collected in a sterile tube, placed immediately on ice, centrifuged at $1,500 \mathrm{rpm}$ for $5 \mathrm{~min}$ to separate the cell contents, then rapidly distributed in two vials, one for VEGF and other for PEDF immediately frozen at $-80^{\circ} \mathrm{C}$ until processed. During vitrectomy membrane peeling was done in the eyes with the $\mathrm{ED} / \mathrm{PDR}$, and these were transported in ice to the lab for the immunohistochemistry (IHC).

Measurement of VEGF and PEDF VEGF levels were measured in vitreous samples by enzyme-linked immunosorbent assay (ELISA) using the Quantikine VEGF assay kit (R\&D systems) and measurement of PEDF by using Chemicon International PEDF sandwich ELISA kit (The ELISA kit and PEDF antibody for immunohistochemistry and Western blot were the generous gift from Dr. Joyce Tombran-Tink, Penn State College of Medicine, Hershey).
IHC for VEGF and PEDF in epiretinal membranes The IHC for VEGF and PEDF was done to confirm the presence of these factors in the epiretinal membrane (ERM) of ED patients at the protein level. The epiretinal/fibrovascular membrane specimen obtained by surgical excision from ED and PDR cases, respectively, were processed for sectioning after fixing in buffered formalin. IHC was done on tissue section of $5 \mu \mathrm{m}$. The deparaffinized sections were incubated with trypsinEDTA for $30 \mathrm{~min}$ and washed with Tris buffered saline ( $\mathrm{pH}$ 7.6). Further steps were carried out with Novolink ${ }^{\mathrm{TM}}$ min polymer detection system (kit from Novacastra laboratories Ltd, NE12 8EW, UK). The slides were blocked for peroxidase with hydrogen peroxide provided in the kit for $10 \mathrm{~min}$, and TBS wash was subsequently given followed by incubation with protein block for $30 \mathrm{~min}$. After this blocking step, slides were incubated for $2 \mathrm{~h}$ with rabbit polyclonal antibody directed against VEGF (Santa Cruz Biotechnology, Santa Cruz, CA, USA) and rabbit polyclonal PEDF. A negative control was done along with, by omitting the primary antibody. These antibodies were characterized as described by the suppliers and in the literature and were used at a dilution of 1:200 for VEGF and 1:150 for PEDF. After washing with TBS, the slides were incubated for $45 \mathrm{~min}$ with post-primary block (Novolink ${ }^{\mathrm{TM}}$ Min polymer detection system). The slides were washed again and incubated for 45 min with polymer link (Novolink ${ }^{\mathrm{TM}}$ Min polymer detection system). Finally, the slides were washed in TBS and rinsed with $0.1 \mathrm{M}$ acetate buffer $\mathrm{pH}$ 5.0-5.2 and stained for $20 \mathrm{~min}$ with $0.8 \%$ Amino ethyl carbazone in acetate buffer ( $\mathrm{pH} 5.0$ ) (Sigma, St Louis, MO, USA) or DAB as the chromogen (Novolink $^{\mathrm{TM}}$ Min polymer detection system). The sections were counterstained with Mayer's haematoxylin for $15 \mathrm{~s}$, rinsed in tap water and air-dried.

Western blot analysis for PEDF Fifty-microgram proteins from the vitreous of ED, PDR, and $\mathrm{MH}$ were loaded on $10 \%$ SDS-PAGE and transblotted to the PVDF membrane.
Table 5 PEDF levels in the vitreous specimen of $\mathrm{ED} / \mathrm{PDR} / \mathrm{MH}$

$\mathrm{PEDF}$ is expressed as Mean $\pm \mathrm{SD}$. $P$ values are expressed based on the comparison of the individual disease with macular hole

\begin{tabular}{llll}
\hline Particulars & Macular hole & Eales disease & PDR \\
\hline No. of specimen & 7 & 26 & 17 \\
Mean age & $59 \pm 10$ & $32 \pm 8$ & $54 \pm 8$ \\
Mean PEDF $(\mu \mathrm{g} / \mathrm{ml})$ & $7.5 \pm 0.7$ & $4.8 \pm 3.9, p=0.027$ & $3.74 \pm 3.80, p=0.008$ \\
Median & 7.3 & 3.9 & 1.8 \\
\hline
\end{tabular}




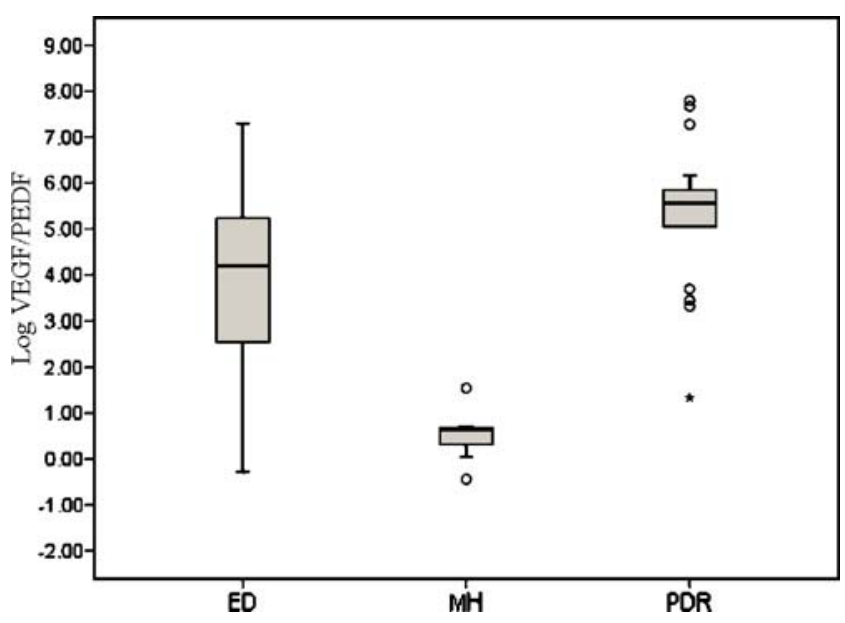

Fig. 1 VEGF/PEDF ratio in ED $(n=26) / \mathrm{MH}(n=27) / \mathrm{PDR}(n=17)$. The $p$ values are given as comparison between $\mathrm{MH}$ (disease control) vs. $\operatorname{ED}(p=0.01) ; \mathrm{MH}$ vs. PDR $(p=0.000)$ and PDR vs. $\operatorname{ED}(p=0.009)$

Pretreatment was done with 5\% nonfat dried milk in $50 \mathrm{mM}$ Tris buffer ( $\mathrm{pH}$ 7.4) followed by incubation for $1 \mathrm{~h}$ with a rabbit polyclonal antibody prepared against human PEDF diluted at 1:3,000 in Tris buffer containing $0.5 \%$ milk. After this treatment, the membranes were washed extensively with Tris buffer and subjected to further incubation for $30 \mathrm{~min}$ in an appropriate dilution of mouse anti-rabbit IgG-HRP. The HRP color development was done by chemiluminescence kit (Millipore).

Statistics The results were analyzed using Mann-Whitney Test and ANOVA. All the statistical analyses were done by SPSS version 14.0.

\section{Results}

Compared to the vitreous of macular hole which showed a mean VEGF level of $14.9 \pm 9.25 \mathrm{pg} / \mathrm{ml}$ in the $\mathrm{ED}$, there was a significant increase to a mean level of $850.5 \pm 1832 \mathrm{pg} / \mathrm{ml}$ $(p=0.000)$. Similarly, in PDR cases, the vitreal VEGF level was significantly increased to $1001.8 \pm 1165.8 \mathrm{pg} / \mathrm{ml}$ ( $p=$ 0.001) compared to disease control namely macular hole. The median shows that the VEGF levels are similar in both PDR and ED groups compared to MH (Table 4). But the levels of PEDF in the vitreous of PDR patients showed a mean level of $3.74 \pm 3.8 \mu \mathrm{g} / \mathrm{ml}$ which is lower than in ED that had a mean level of $4.8 \pm 3.9 \mu \mathrm{g} / \mathrm{ml}$ compared to macular hole with a mean PEDF level of $7.5 \pm 0.7 \mu \mathrm{g} / \mathrm{ml}$, (PDR vs. MH: $p=0.027$ and ED vs. MH: $p=0.008$ ). The median is lower in PDR patients compared to ED cases, though in both groups, it is lower than MH (Table 5). The ratio of VEGF to PEDF was found to be significantly increased in $\mathrm{ED}$ (ratio $=151 \pm 304 ; p=0.01$ ) and PDR (ratio= $528 \pm 742 ; p=0.000$ ) compared to the macular hole (ratio= $2.01 \pm 1.2$ ). However, the ratio was 3.5 -fold higher in PDR than ED, and this ratio is significantly higher in PDR than in $\mathrm{ED}$ ( $p=0.009$; Fig. 1).

The high angiogenic potential is seen as the ratio of VEGF/PEDF correlates with the peak clinical onset of the ED in the age group 21-30 years, and the diseases usually self-resolves above the age of 40 , which is reflected by the low ratio of VEGF/PEDF (Table 6).

The relation between laser treatment and the levels of VEGF and PEDF in the vitreous of ED patients is shown in Table 7. The mean VEGF levels were found to be significantly elevated in the sectoral laser treated group than the "no laser" group (patients who did not warrant laser treatment; $p=0.026$ ). The PEDF levels and the VEGF/ PEDF ratio too were elevated but were not statistically significant. Owing to the small sample size, the distribution profile was made, which shows that the VEGF levels are distinctly clustered at relatively lower levels in the "no laser" group compared to both sectoral and PRP. But in the PDR cases, the "no laser"-treated group (who did not warrant laser treatment) had VEGF level (mean and the median) higher than the laser-treated group.

The ED patients under no steroid treatment have lower VEGF/PEDF ratio in terms of both mean and the median, though it was not statistically significant. However, the
Table 6 Relation between age and VEGF, PEDF levels in the vitreous of ED patients

\begin{tabular}{|c|c|c|c|c|c|}
\hline Age group (year) & No. of Patients & Parameter & VEGF pg/ml & PEDF $\mu \mathrm{g} / \mathrm{ml}$ & Ratio \\
\hline$\leq 20$ & 2 & Mean & $1,701 \pm 567$ & $5.7 \pm 8$ & $92.2 \pm 130$ \\
\hline \multirow[t]{2}{*}{$21-30$} & \multirow[t]{2}{*}{8} & Mean & $2,064 \pm 2,975$ & $4.86 \pm 3.5$ & $371 \pm 475$ \\
\hline & & Median & 598 & 3.9 & 214 \\
\hline \multirow[t]{2}{*}{$31-40$} & \multirow[t]{2}{*}{13} & Mean & $157 \pm 143$ & $4.86 \pm 4.1$ & $58.9 \pm 111$ \\
\hline & & Median & 102 & 3.5 & 19.4 \\
\hline \multirow[t]{2}{*}{$>40$} & \multirow[t]{2}{*}{3} & Mean & $50.1 \pm 14$ & $3.63 \pm 3.1$ & $6.6 \pm 6$ \\
\hline & & Median & 43 & 5.4 & 7.3 \\
\hline \multicolumn{6}{|l|}{$P$ value } \\
\hline \multicolumn{2}{|c|}{$21-30$ vs. $31-40$ years } & & 0.03 & NS & 0.03 \\
\hline \multicolumn{2}{|c|}{$21-30$ vs. $>40$ years } & & 0.28 & NS & 0.23 \\
\hline
\end{tabular}


Table 7 Relation between Laser treatment and VEGF, PEDF levels in the vitreous of ED patients

\begin{tabular}{llllll}
\hline Group & No. of patients & Parameter & VEGF pg/ml & PEDF $\mu \mathrm{g} / \mathrm{ml}$ & Ratio \\
\hline No Laser & \multirow{2}{*}{12} & Mean & $265 \pm 297$ & $4.28 \pm 3.9$ & $98 \pm 132$ \\
& \multirow{2}{*}{4} & Median & 88 & 3.6 & 20 \\
Sectoral Phc & 4 & Mean & $2304 \pm 3034$ & $6.85 \pm 3.6$ & $444 \pm 693$ \\
& & Median & 1230 & 6.25 & 143 \\
PRP & \multirow{2}{*}{10} & Mean & $971 \pm 2176$ & $4.56 \pm 4.2$ & $98.6 \pm 176$ \\
& & Median & 217 & 4.7 & 33.4 \\
Paired $t$ test & & & & 0.100 \\
No laser vs. Sectoral Phc & $p$ value & 0.026 & 0.265 & 0.994 \\
No laser vs. PRP & $p$ value & 0.277 & 0.873 & 0.148 \\
Sectoral Phc vs. PRP & $p$ value & 0.370 & 0.359 & \\
\hline
\end{tabular}

distribution shows that the steroid-treated group has relatively higher ratio and was maximum in the group treated with both oral and periocular steroids (Table 8). This can possibly be explained by the fact that clinically, the treatment is guided by the severity of disease.

Both VEGF and PEDF were detected in the ERM specimen from the ED cases as well as in the fibrovascular membrane from the PDR case (Fig. 2).

The Western blot analysis of the vitreous specimen for PEDF in ED compared to the PDR and macular hole is shown in Fig. 3. The densitometry analysis showed that the band intensity of the PDR is the lowest followed by ED and macular hole compared to the donor eye vitreous.

\section{Discussion}

Ocular neovascularization is regulated by a diffusible factor, VEGF that promotes angiogenesis in the retina which is known to play a role in the pathogenesis of early diabetic retinopathy [14]. In addition, lower levels of pigment epithelium-derived factor have been related to the angiogenesis in diabetic retinopathy that results in active proliferative diabetic retinopathy [15]. It is also well documented that advanced glycation end products (AGE)- advanced glycation end product receptor (RAGE) interaction elicited angiogenesis through the transcriptional activation of the VEGF gene [16]. Interaction of AGEs with RAGE leads to leukostasis and blood-retinal barrier breakdown that are characteristic findings in diabetic retinopathy [17]. While AGEs in the form of methylglyoxal detected in diabetic membrane and serum suggest the role of glycation. Results from our previous studies suggest that AGEs formed through glycoxidation may play an important role in the development of retinal neovascularization seen in ED. The immunoreactivity of CML-AGEs (carboxy methyl lysine) in neovascular membrane and its increased levels in serum suggest that in spite of the normoglycemic status, the glycoxidation resulting in AGE and oxidative stress may trigger the retinal neovascularization in ED. Therefore, this study was aimed at estimating the VEGF and PEDF levels in the vitreous specimen of ED and to see the ratio in comparison with PDR. Present study shows that the mean VEGF level in the vitreous is significantly increased in $\mathrm{ED}$ compared to $\mathrm{MH}$ and the distribution is similar to that seen in the PDR cases. Ninety-eight percent of the ED patients and $96 \%$ of the PDR cases showed VEGF levels over and above the median level of the MH group. The increased levels of VEGF might explain the severity of the neovascularization and hemorrhage in the
Table 8 Relation between steroid intake and the VEGF, PEDF levels

\begin{tabular}{llllll}
\hline Group & No. of Patients & Parameter & VEGF & PEDF & Ratio \\
\hline No steroid & 9 & Mean & $222 \pm 279$ & $3.98 \pm 3.6$ & $71.7 \pm 92.7$ \\
& & Median & 102 & 308 & 12.8 \\
Oral steroids & 11 & Mean & $842 \pm 2,073$ & 6.15 & 125 \\
& & Median & 325 & 5.5 & 36.1 \\
Oral + periocular steroids & 6 & Mean & $1,807 \pm 2,501$ & $3.48 \pm 4.3$ & $320 \pm 576$ \\
& & Median & 986.5 & 2.5 & 108.6 \\
Paired $t$ test & & & & \\
No steroids vs. oral steroids & & & 0.21 & 0.44 \\
No steroid vs. oral + periocular steroids & $p$ value & 0.38 & 0.81 & 0.21 \\
Oral steroids vs. oral + periocular steroids & $p$ value & 0.40 & 0.21 & 0.31 \\
\hline
\end{tabular}



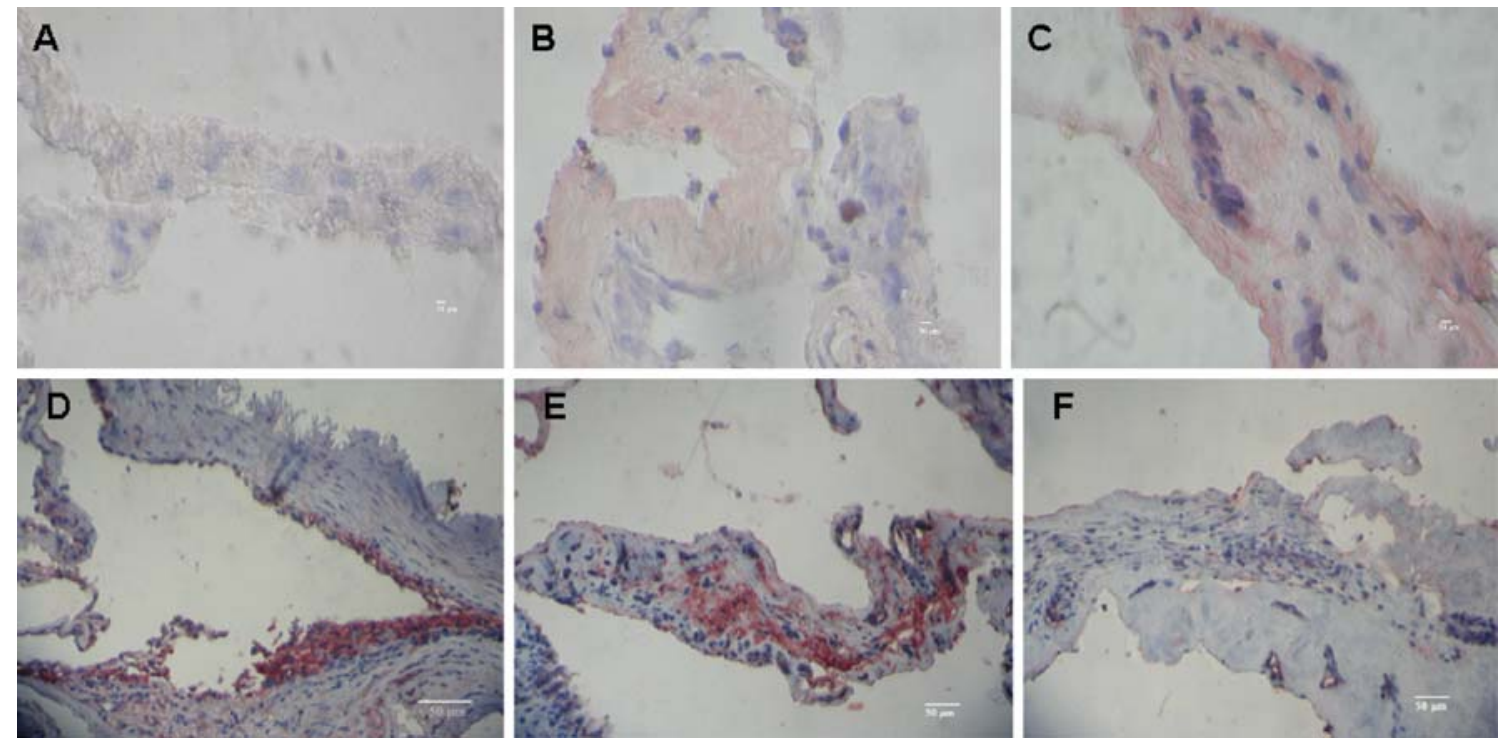

Fig. 2 A-C VEGF immunostaining in epiretinal membrane of ED and PDR is visualized with a red reaction product. A ERM from a patient with ED negative for secondary antibody; B ERM from ED patient showing VEGF posivity; C ERM from PDR patient showing VEGF positivity. D-F PEDF immunostaining in epiretinal membrane

$\mathrm{ED}$, similar to the report on PDR wherein studies have shown that high levels of VEGF accumulate in the vitreous of patients with proliferative diabetic retinopathy, which results in the mitotic effect on the retinal capillary endothelial cells [18]. The report by Kumar and Sinha [19] on intravitreal bevacizumab in ED has been indicative of role of VEGF in the neovascularization seen in ED. However, there has been no report on the vitreous levels of VEGF in the ED cases. The intraocular synthesis of angiogenic factors is counterbalanced by the synthesis of antiangiogenic factors. The main antiangiogenic factor is the PEDF though there are also factors such as transforming growth factor beta, thrombospondin, and somatostatin [20]. The study revealed that there is a significant decrease in the PEDF levels in the vitreous of ED cases. Seventy percent of ED cases show lowered PEDF levels compared to the median level of $\mathrm{MH}$, and in the PDR group, it is $82 \%$. PEDF has been proposed as a therapeutic target for

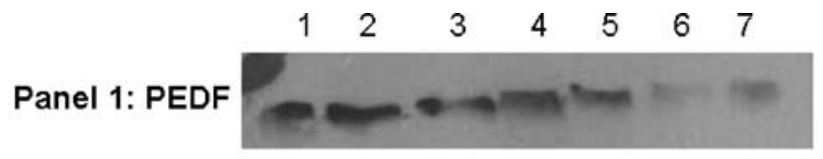

\section{Panel 2: $\beta$ actin}

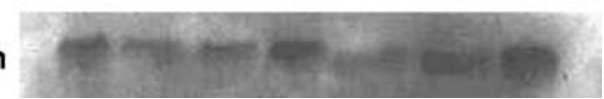

Fig. 3 Western blot analysis of the vitreous specimen for PEDF in Eales' disease compared to the PDR and MH. Panel 1: Western blot analysis of vitreous PEDF in ED, PDR, MH, and donor eye vitreous. Panel 2: Beta Actin. Lane 1 donor eye vitreous, Lane $2 \mathrm{MH}$, Lane 3 $\mathrm{ED}$, Lane $4 \mathrm{ED}$, Lane $5 \mathrm{PDR}$, Lane $6 \mathrm{PDR}$, Lane $7 \mathrm{PDR}$ of ED, and PDR is visualized with a red reaction product. D ERM from ED patient showing PEDF positivity; E ERM from PDR patient showing PEDF positivity; F ERM from a patient with ED negative for secondary antibody

oxidative stress-involved eye diseases, such as PDR [21]. Moreover, it is also earlier reported as an anti-inflammatory factor that can inhibit VEGF-induced vascular permeability [22]. In this study, the IHC shows the VEGF and PEDF at the protein level in the ERM of the ED cases which is comparable to that of PDR.

The balance between the angiogenic and anti-angiogenic factors rather than angiogenic factors themselves is said to be crucial in determining the progression of the angiogenic vitreoretinopathies such as PDR. Therefore, the ratio of VEGF/PEDF was analyzed, and the study revealed that the PDR had the highest ratio followed by ED and the desirable levels were seen in MH. The ratio was significantly higher in PDR than in the ED. Higher VEGF/PEDF index values have been associated with the higher angiogenic potential [23]. Although VEGF was raised in both diseases, PEDF was found to be more lowered in the PDR than in ED. But
Fig. 4 Neovascularization mechanism in Eales' disease $\uparrow$ ROS/RNS

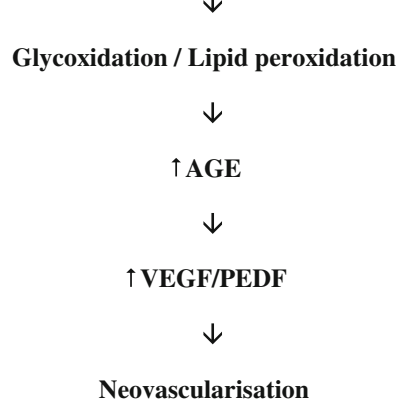

Neovascularisation 
this was not statistically significant. However, the ratio gives a clear picture of the angiogenic potential that was significantly higher in PDR compared to ED. Clinically, ED is a selflimiting disease while PDR is a progressive disease.

While looking at the effect of steroid on VEGF, PEDF levels, and VEGF/PEDF ratio in the ED cases, a subgroup analysis of the interval between last steroid intake and time of vitrectomy showed an observation that the VEGF levels and the VEGF/PEDF ratio were lower in those receiving steroids between 1 and 6 months preceding surgery as compared to those receiving steroids in the month immediately preceding surgery. Although multiple factors can influence the instantaneous levels of VEGF and PEDF in the vitreous, the treatment with effect to time lag can explain the above observation. Similarly, the interval between last laser treatment and time of vitrectomy was looked into. However, no particular trend was noted here, and no statistical difference was noted in VEGF, PEDF, and VEGF/PEDF ratio between the groups (data not shown). The VEGF levels in the sectoral laser treatment were found to be significantly higher than the "no laser" group, and the ratio of VEGF/PEDF was also higher in spite of the increased PEDF levels. The sectoral laser is done when the disease is relatively still active while in the PRP the disease is in the involuntary stage. The PRP group showed the ratio similar to the "no laser" group. However, the sample sizes have to be considerably high before drawing any conclusions. Funatsu et al. have shown a correlation between the VEGF levels and the grade of photocoagulation [24]. In the PDR patients, the laser-treated group showed lower angiogenic potential than the no laser group. Vitreous VEGF levels are reportedly higher in PDR patients with no laser treatment than the PDR patients treated with laser. How little the laser treatment is and the timely treatment can possibly influence the VEGF and the PEDF levels.

The earlier study from this laboratory has shown the CML-AGE immunoreactivity detected in all cases of ED and $61 \%$ cases of diabetic retinopathy and none in idiopathic ERM [25]. AGE accumulation seen also in the serum of these patients inspite of normoglycemia as against diabetes was explained based on the glycoxidation process in ED triggered by the iron-induced oxidative stress [26]. Also, increasing evidence indicates that AGEs promote retinal alterations through oxidative stress [27, 28]. Though ageing and hyperglycemia as in ARMD and diabetic retinopathy, respectively, can result in AGE formation, in $\mathrm{ED}$, it is very clear that oxidative stress and nitrosative stress precedes AGE accumulation [6, 29].

The angiogenesis is inhibited normally by inherent ocular inhibitor-PEDF. Low levels of PEDF along with increased VEGF has a net angiogenic effect that result in the neovascularization seen in ED. Oxidative stress may involve in retinal PEDF downregulation [30]. Further,
PEDF is known to inhibit AGE-induced VEGF expression [31]. Thus, in ED, increased oxidative stress leads to AGE formation owing to glycoxidation, which is followed by AGE-RAGE-mediated VEGF elaborated (Fig. 4). Inflammation, NV, and retinal damage in ED could be explained in terms of accumulation of lipid and oxygen-free radicals caused by neovascularization. This is further upregulated by the low levels of PEDF. Intraocular neovascularization develops in numerous ischemic retinal vein disorders. In proliferative diabetic retinopathy, there is active and extensive proliferation of new vessels that leads to vitreous hemorrhage and retinal detachment. Though ED is not exactly similar to diabetic retinopathy wherein the disease is continuous and ongoing process, in ED, there is an initial insult that may end up with the proliferative phase. The management depends on the stage of the disease and consists of (a) treatment with oral corticosteroids in the active inflammatory stage and (b) laser photocoagulation in the neovascularization state and vitrectomy when there is vitreous hemorrhage or retinal detachment. The relapse and remission are characteristic of Eales disease unlike Proliferative diabetic retinopathy.

Open Access This article is distributed under the terms of the Creative Commons Attribution Noncommercial License which permits any noncommercial use, distribution, and reproduction in any medium, provided the original author(s) and source are credited.

\section{References}

1. Biswas J, Sharma T, Gopal L. Eales' Disease. Surv Ophthalmol 2002;47:197-214.

2. Ramakrishnan S, Rajesh M, Sulochana KN. Eales' disease: Oxidant stress and weak antioxidant defence. Indian J Ophthalmol 2007;55:95-102.

3. Gieser SC, Murphy RP. Eales' disease. In: Ryan SJ, editor. Retina Vol II. Medical Retina. St. Louis: CV Mosby; 1994. p. 1503-7.

4. Das T, Biswas J, Kumar A, et al. Eales' disease. Indian J Ophthalmol 1994;42:3-18.

5. Saxena S, Kumar D. A new staging system of idiopathic retinal periphlebitis. Eur J Ophthalmol 2004;14:236-9.

6. Rajesh M, Sulochana KN, Punitham R, Lakshmi S, Ramakrishnan $\mathrm{S}$. Involvement of oxidative and nitrosative stress in promoting retinal vacuities in patients with Eales' disease. Clin. Biochem 2003;36(5):377-85.

7. Rajesh M, Sulochana KN, Coral K, et al. etermination of carbonyl groups content in plasma proteins as a useful marker for assessing the impairment in antioxidant defense in patients' with eales' disease. Indian J Ophthalmol 2004;52:139-44.

8. Rajesh M, Sulochana KN, Ramakrishnan S, Biswas J, Manoharan PT. Iron chelation abrogates excessive formation of hydroxyl radicals and lipid peroxidation products in monocytes of patients' with Eales' disease: direct evidence using electron spin resonance spectroscopy. Curr Eye Res 2004;28(6):399-407.

9. Aiello LP, Avery RL, Arrigg PG, et al. Vascular endothelial growth factor in ocular fluid of patients with diabetic retinopathy and other retinal disorders. N Engl J Med 1994;331:1480-7.

10. Ogata N, Tombran-tink J, Nishikawa M, et al. Pigment epitheliumderived factor in the vitreous is low in diabetic retinopathy and 
high in rhegmatogenous retinal detachment. Am J Opthalmol 2001;132:378-82.

11. Boehm BO, Lang G, Volpert $\mathrm{O}$, et al. Low content of the natural ocular anti-angiogenic agent pigment epithelium-derived factor (PEDF) in aqueous humor predicts progression of diabetic retinopathy. Diabetologia 2003;46:394-400.

12. Ho AC, Guyer DR, Fine SL. Macular hole. Surv Ophthalmol 1998;42:393-416.

13. Ezra E. Idiopathic full thickness macular hole: natural history and pathogenesis. Br J Ophthalmol 2001;85:102-8.

14. Ishida $\mathrm{S}$, Usui T, Yamashiro K, et al. VEGF164 is proinflammatory in the diabetic retina. Invest Ophthalmol Vis Sci 2003;44:2155-62.

15. Ogata N, Wada M, Otsuji T, Jo N, Tombran-Tink J, Matsumura M. Expression of pigment epithelium-derived factor in normal adult rat eye and experimental choroidal neovascularization. Invest Ophthalmol Vis Sci 2002;43:1168-75.

16. Yamagishi S, Yonekura H, Yamamoto Y, Katsuno K, Sato F, Mita I. Advanced glycation end products-driven angiogenesis in vitro induction of the growth and tube formation of human microvascular endothelial cells through autocrine vascular endothelial growth factor. J Biol Chem 1997;272:8723-30.

17. Kaji Y, Usui $T$, Ishida $S$, et al. Inhibition of Diabetic Leukostasis and Blood-Retinal Barrier Breakdown with a Soluble Form of a Receptor for Advanced Glycation End Products. Invest Ophthalmol Vis Sci 2007;48:858-65.

18. Patel JI, Tombran-Tink J, Hykin PG, et al. Vitreous and aqueous concentrations of proangiogenic, antiangiogenic factors and other cytokines in diabetic retinopathy patients with macular edema: Implications for structural differences in macular profiles. Exp Eye Res 2006;82:798-806.

19. Kumar A, Sinha S. Rapid regression of disc and retinal neovascularization in a case of Eales disease after intravitreal bevacizumab. Can J Ophthalmol 2007;42:335-6.

20. Carrasco E, Andez CH, Miralles A. Lower somatostatin expression is an early event in diabetic retinopathy and is associated with retinal neurodegeneration. Diabetes Care 2007;30:2902-8.

21. Yoshida Y, Yamagishi S, Matsui T. Positive correlation of pigment epithelium-derived factor and total antioxidant capacity in aqueous humour of patients with uveitis and proliferative diabetic retinopathy. Br J Ophthalmol 2007;91:1133-4.
22. Zhang SX, Wang JJ, Gao G, Shao C, Mott R, Ma JX. Pigment epithelium-derived factor (PEDF) is an endogenous antiinflammatory factor. FASEB J 2006;20:323-5.

23. Agnieszka KB, Maria HN. Influence of retinal photocoagulation in patients with proliferative diabetic retinopathy on vitreous vascular endothelial growth factor concentration. Diabetologia 2007;7(2):97-102.

24. Funatsu H, Yamashita H, Noma H. Outcome of vitreous surgery and the balance between vascular endothelial growth factor and endostatin. Invest Ophthalmol Vis Sci 2003;44:1042-7.

25. Swamy-Mruthinti S, Miriam KC, Krishnakumar S, et al. Immunolocalization and quantification of advanced glycation end products in retinal neovascular membranes and serum: A possible role in ocular neovascularization. Curr Eye Res 2002;25:139-45.

26. Sulochana KN, Coral K, Punitham R. Trace elements iron, copper and zinc in vitreous of patients with various vitreoretinal diseases. Indian J Opthalmol 2004;52:145-8.

27. Li L, Renier G. Activation of nicotinamide adenine dinucleotide phosphate (reduced form) oxidase by advanced glycation end products links oxidative stress to altered retinal vascular endothelial growth factor expression. Metabolism 2006;55:1516-23.

28. Yamagishi S, Imaizumi T. Molecular mechanism of diabetic retinopathy: role of advanced glycation end products (AGEs) and their receptor (RAGE) in the pathogenesis of diabetic retinopathy. Nippon Ganka Gakkai Zasshi 2005;109:338-45.

29. Yokoi M, Yamagishi SI, Takeuchi M. Elevations of AGE and vascular endothelial growth factor with decreased total antioxidant status in the vitreous fluid of diabetic patients with retinopathy. $\mathrm{Br}$ J Ophthalmol 2005;89:673-5.

30. Saito W, Hirose S, Ohgami K, Kase M, et al. Positive association of pigment epithelium-derived factor (PEDF) with total antioxidant capacity in the vitreous fluid of patients with proliferative diabetic retinopathy. Br J Ophthalmol 2007;7:1-9.

31. Yamagishi S, Nakamura $K$, Matsui $T$, et al. Pigment epithelium-derived factor (PEDF) inhibits advanced glycation end product-induced retinal vascular hyperpermeability by blocking reactive oxygen species-mediated vascular endothelial growth factor (VEGF) expression. J Biol Chem 2006;281:2021320. 